\title{
Modeling of All-Optical Even and Odd Parity Generator Circuits Using Metal-Insulator-Metal Plasmonic Waveguides
}

\author{
Lokendra SINGH, Amna BEDI, and Santosh KUMAR* \\ Photonics Lab, Department of Electronics and Communication Engineering, DIT University, Dehradun-248009, India \\ *Corresponding author: Santosh KUMAR？ E-mail: santoshrus@yahoo.com
}

\begin{abstract}
Plasmonic metal-insulator-metal (MIM) waveguides sustain excellent property of confining the surface plasmons up to a deep subwavelength scale. In this paper, linear and S-shaped MIM waveguides are cascaded together to design the model of Mach-Zehnder interferometer (MZI). Nonlinear material has been used for switching of light across its output ports. The structures of even and odd parity generators are projected by cascading the MZIs. Parity generator and checker circuit are used for error correction and detection in an optical communication system. Study and analysis of proposed designs are carried out by using the MATLAB simulation and finite-differencetime-domain (FDTD) method.
\end{abstract}

Keywords: Plasmonics; MIM waveguides; Mach-Zehnder interferometer; nonlinear process; FDTD method

Citation: Lokendra SINGH, Amna BEDI, and Santosh KUMAR, "Modeling of All-Optical Even and Odd Parity Generator Circuits Using Metal-Insulator-Metal Plasmonic Waveguides,” Photonic Sensors, 2017, 7(2): 182-192.

\section{Introduction}

In today's scenario of photonic industry, the major challenge is the miniaturization of optical components for wavelength division multiplexing [1]. Despite the deep subwavelength confinement of surface plasmon polaritons (SPPs) with respect to other nanophotonic waveguides such as photonic crystals, SPP waveguides work well beyond the diffraction limit [2-4]. Plasmonics is one of the promising fields of integrated photonics due to its numerous applications in optical clocking, cancer therapy, nano-lithography, solar cells, etc. In designing of building blocks for future generation of integrated optical components and devices, plasmonic waveguides play a significant role. Recently, several types of waveguides were discovered such as slots $[5,6]$, plasmonic wedge waveguide [7], and dielectric ridge on the metal surface [8], but among all these waveguides the metal-insulator-metal (MIM) waveguide is generally preferred due to its their exceptional property of confining the surface plasmons to a deep subwavelength scale [9-13]. In these waveguides, propagation is achieved by using even modes due to their low-loss confinement profile. Considering the photonic device altogether, directional couplers (DCs) are serves as a prime component to design the elementary part of optical circuits. Recently, several designs of directional couplers have been proposed by using plasmonic waveguides [14-17]. The major phenomenon of coupling of light within the DC is beating of even-odd modes. As the equal splitting of power through the directional coupler is wavelength dependent, for broadband response either wavelength dependency should be removed by

Received: 20 July 2016 / Revised: 16 November 2016

(C) The Author(s) 2017. This article is published with open access at Springerlink.com

DOI: $10.1007 / \mathrm{s} 13320-017-0365-9$

Article type: Regular 
making coupler adiabtic, or input power with its coupling length should be controlled. In the proposed structure of DC connected in the Mach-Zehnder interferometer (MZI), equal splitting of power is attained with coupling length and propagation length of $1 \mu \mathrm{m}$ and $10.2 \mu \mathrm{m}$, respectively. Hence, by adjoining the two DCs using two linear MIM waveguides, a design of MZI has been designed within the footprints of $(16.2 \times 4) \mu \mathrm{m}$. Although interferometric circuit using Fabry-Perot interferometer is also used in various applications, the need of too many sources will lead to an increase in the cost and complexity of devices [18]. One of the arms of MZI is filled with an active material. Here, MEH-PPV [poly (2-methoxy-5(28-ethylexyloxy)-PPV)] is used as an active material with refractive index and response time of $n=1.65$ and $\tau=2.1 \mathrm{e}^{-15} \mathrm{~s}$, respectively [19]. The presence of active materials in the structure of MZI is used to switch the light across its output ports by changing the refractive index of waveguide with respect to power of input signals. The changes in phase canceling out or adding each other depend on nonlinear processes. If phase of both the signals cancels out each other then it crosses phase modulation (XPM), and if it adds then it is self phase modulation (SPM). Due to XPM, the optical signal arrives at through port and SPM thrives the signal at cross port of MZI. Thus, by cascading plasmonic DCs and MZIs, all-optical structures of even and odd parity generators are modeled within the footprints of $(125 \times 10) \mu \mathrm{m}$ and $(205 \times 10) \mu \mathrm{m}$, respectively. Recently, some switching circuits have been proposed by using $\mathrm{LiNbO}_{3}$-based MZI, which work on the principle of electro-optic effect [20]. Although the work is excellent, it still lacks advanced applications in terms of device miniaturization up to a deep subwavelength scale (hence overall design of device remains in thousand of micrometers), and due to the presence of electrical signals for altering the refractive index of waveguides, it limits the speed of device. Some switching circuits were also proposed by using semiconductor optical amplifier (SOA) but they were backsitted due to the phenomenon of gain saturation [21]. All optical logic gates were also proposed using photonic crystal but being lack of the nonlinear material inside the structure will enable the switching of optical signals for complex circuits [22].

The proposed design of parity generators are verified by using the finite difference time domain (FDTD) method [23] and MATLAB simulation. Section 2 presents the models of even and odd parity generators with their mathematical formulations. In Section 3, the propagation of light through proposed structures is projected. Finally, the conclusions of complete work are presented in Section 4.

\section{Design of parity generator circuits}

The combinational circuits are useful in designing various integrated circuits which are very useful in micro-controlling and central processing units. In this work, for designing the all optical circuits of parity generators, MIM plasmonic waveguides are used. To create the MIM geometry, a metal (SiON) having high refractive index of $n_{1}=2.01$ with permittivity of $-1+\mathrm{j} 2.9$ is deposited over a low index substrate having refractive index of $n_{2}=1.44$, and air is considered as dielectric between them. The thickness of dielectric is taken as $0.5 \mu \mathrm{m}$ for the propagation of surface plasmons with a propagation length of $10.2 \mu \mathrm{m}$. Thus by using these MIM waveguides, the structure of MZI is designed as shown in Fig. 1, whose second linear arm is filled with nonlinear Kerr or active material. When the optical signals with power of $E_{\text {in }}=0.014 \mathrm{~W} / \mu \mathrm{m}$ (considered as digital logic " 0 ") are given at the first input port, then by obeying the phenomenon of SPM, signals are obtained at the second output port of MZI as shown in Fig. 2(a). When signals with the power $E_{\text {in }}=0.081 \mathrm{~W} / \mu \mathrm{m}$ (considered as digital logic "1") are incident at the first input port of MZI, then 
due to XPM optical signals arrive at the first output port of it as shown in Fig. 2(b). The extinction ratio (ER) of single MZI is about $26 \mathrm{~dB}$ at output power difference of $0.7 \mathrm{~dB}$ and $0.8 \mathrm{~dB}$. The analyzed value of ER for single MZI is quite useful and enough for designing the all-optical logic gates [24].

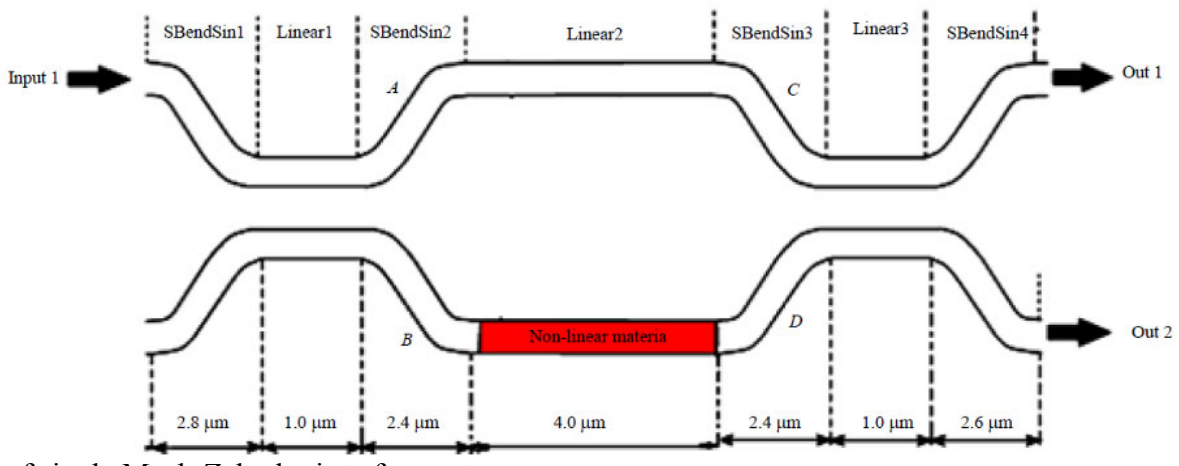

Fig. 1 Schematic of single Mach-Zehnder interferometer.

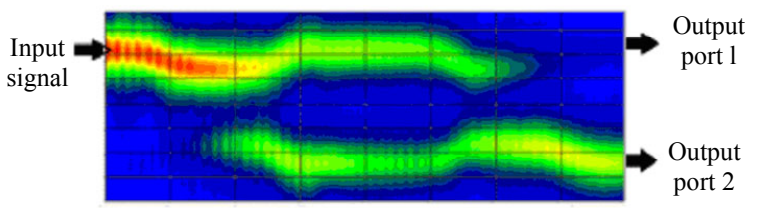

(a)

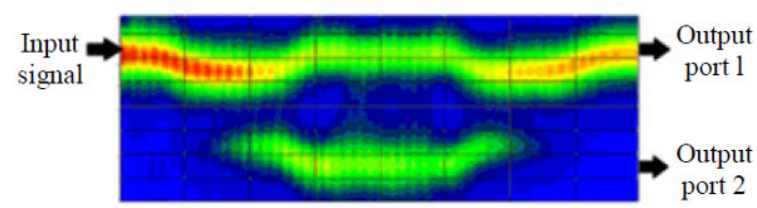

(b)

Fig. 2 Propagation of light through single MZI: (a) for low intensity signals and (b) for high intensity signals.

Thus, this toggling of optical signals across output ports of MZI with respect to input power of source is mathematically written as [25-28]

$$
\begin{aligned}
& T_{\text {out } 1}=\sin ^{2}\left(\frac{\Delta \varphi}{2}\right) a t E_{\text {in }}=0.014 \mathrm{~W} / \mu \mathrm{m} \\
& T_{\text {out2 }}=\cos ^{2}\left(\frac{\Delta \varphi}{2}\right) a t E_{\text {in }}=0.081 \mathrm{~W} / \mu \mathrm{m}
\end{aligned}
$$

where $\Delta \varphi$ is the total change in phase in two linear arms of MZI. Equations (1) and (2) represent the mathematical expressions for outputs of MZI when input is fed with high and low powers, respectively.

\subsection{Even parity generator}

Figure 3 shows the digital circuit and K-map of three bit even parity generators, and Table 1 presents the truth table of the even parity generator. The circuit of the even parity generator is designed by cascading six MZIs and four directional couplers as shown in Fig. 4. For the proper functioning of device, three optical sources are placed in front of the second input ports of MZI1, MZI2, and MZI3. Three directional couplers DC1, DC2, and DC3 for splitting the optical energy into two equal parts are connected at the second output port of all three MZIs, respectively. The second output ports of DC1 and DC2 are combined together with the first output port of DC3 to feed the MZI5. While the first output ports of DC1 and DC2 are combined together to feed the MZI4 at the second input port DC4 is connected at the second output port of same MZI. Then the second output ports of DC3 and DC4 are combined together to feed MZI6 at its first input port. Finally, the second and first output ports of MZI5 and MZI6 are combined together to get the required output of even parity generator.
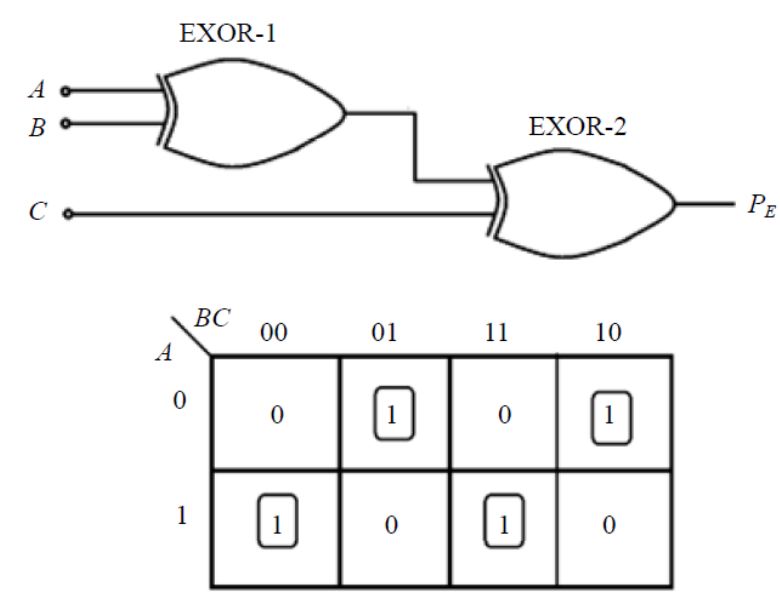

$P_{E}=A \oplus B \oplus C$

Fig. 3 Digital circuit and K-map of three bit even parity generator circuit. 


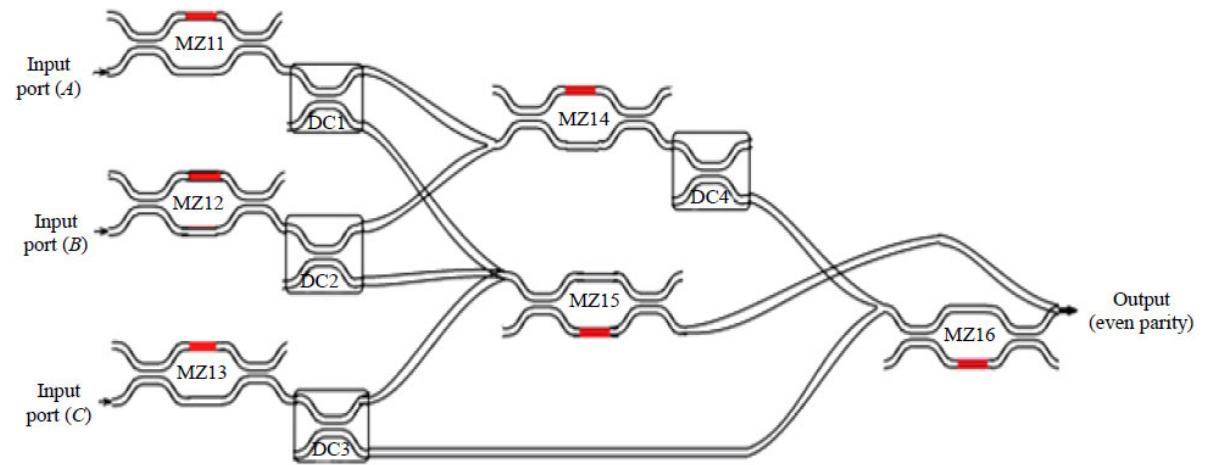

Fig. 4 Schematic diagram of even parity generator.

Table 1 Truth table of even parity generator.

\begin{tabular}{ccccc}
\hline \multirow{2}{*}{ Min-terms } & \multicolumn{3}{c}{ Input signals } & Output signals \\
\cline { 2 - 5 } & $A$ & $B$ & $C$ & $P_{E}$ \\
\hline$m_{1}$ & 0 & 0 & 0 & 0 \\
$m_{2}$ & 0 & 0 & 1 & 1 \\
$m_{3}$ & 0 & 1 & 0 & 1 \\
$m_{4}$ & 0 & 1 & 1 & 0 \\
$m_{5}$ & 1 & 0 & 0 & 1 \\
$m_{6}$ & 1 & 0 & 1 & 0 \\
$m_{7}$ & 1 & 1 & 0 & 0 \\
$m_{8}$ & 1 & 1 & 1 & 1 \\
\hline
\end{tabular}

In Fig. 1, the power at the output of directional coupler towards the input side can be written as [24]

$$
\begin{aligned}
& P_{A}=\sin ^{2}(\alpha L) \\
& P_{B}=\cos ^{2}(\alpha L)
\end{aligned}
$$

where $\alpha$ is the attenuation constant of DC towards input side, and $L$ is its coupling length which is $1 \mu \mathrm{m}$. Thus, by using (1)-(3), mathematical expressions for the output of even parity generator circuit can be written as

$$
\begin{aligned}
m_{1}= & \left.\cos ^{2}\left(\frac{\Delta \varphi_{\mathrm{MZI1}}}{2}\right)+\cos ^{2}\left(\frac{\Delta \varphi_{\mathrm{MZI2}}}{2}\right)+\cos ^{2}\left(\frac{\Delta \varphi_{\mathrm{MZI3}}}{2}\right)\right\} \\
m_{2}= & \cos ^{2}\left(\frac{\Delta \varphi_{\mathrm{MZI1}}}{2}\right)+\cos ^{2}\left(\frac{\Delta \varphi_{\mathrm{MZI2}}}{2}\right)+ \\
& \sin ^{2}\left(\frac{\Delta \varphi_{\mathrm{MZI} 3}}{2}\right) \sin ^{2}\left(\alpha L_{\mathrm{DC} 3}\right) \cos ^{2}\left(\frac{\Delta \varphi_{\mathrm{MZIS}}}{2}\right) \\
m_{3}= & \cos ^{2}\left(\frac{\Delta \varphi_{\mathrm{MZI1}}}{2}\right)+\sin ^{2}\left(\frac{\Delta \varphi_{\mathrm{MZI2}}}{2}\right) \cos ^{2}\left(\alpha L_{\mathrm{DC} 2}\right) \times \\
& \cos ^{2}\left(\frac{\Delta \varphi_{\mathrm{MZI5}}}{2}\right)+\cos ^{2}\left(\frac{\Delta \varphi_{\mathrm{MZI3}}}{2}\right) \\
m_{4}= & \cos ^{2}\left(\frac{\Delta \varphi_{\mathrm{MZI1}}}{2}\right)+
\end{aligned}
$$

$$
\begin{aligned}
m_{6}= & \left\{\begin{array}{l}
\sin ^{2}\left(\frac{\Delta \varphi_{\mathrm{MZI1}}}{2}\right) \cos ^{2}\left(\alpha L_{\mathrm{DC} 1}\right)+ \\
\sin ^{2}\left(\frac{\Delta \varphi_{\mathrm{MZ13}}}{2}\right) \sin ^{2}\left(\alpha L_{\mathrm{DC} 3}\right)
\end{array}\right\} \sin ^{2}\left(\frac{\Delta \varphi_{\mathrm{MZIS}}}{2}\right)+ \\
& \cos ^{2}\left(\frac{\Delta \varphi_{\mathrm{MZ12}}}{2}\right)
\end{aligned}
$$$$
\begin{aligned}
& m_{7}=\left.\begin{array}{l}
\sin ^{2}\left(\frac{\Delta \varphi_{\mathrm{MZI1}}}{2}\right) \cos ^{2}\left(\alpha L_{\mathrm{DC} 1}\right)+ \\
\sin ^{2}\left(\frac{\Delta \varphi_{\mathrm{MZI2}}}{2}\right) \cos ^{2}\left(\alpha L_{\mathrm{DC} 2}\right)
\end{array}\right\} \sin ^{2}\left(\frac{\Delta \varphi_{\mathrm{MZI5}}}{2}\right)+ \\
& \cos ^{2}\left(\frac{\Delta \varphi_{\mathrm{MZI3}}}{2}\right)
\end{aligned}
$$$$
m_{8}=\left[\begin{array}{l}
\sin ^{2}\left(\frac{\Delta \varphi_{\mathrm{MZI1}}}{2}\right) \sin ^{2}\left(\alpha L_{\mathrm{DC} 1}\right)+ \\
\sin ^{2}\left(\frac{\Delta \varphi_{\mathrm{MZI2}}}{2}\right) \sin ^{2}\left(\alpha L_{\mathrm{DC} 2}\right)
\end{array}\right\} \times
$$$$
\left.\begin{array}{l}
\sin ^{2}\left(\frac{\Delta \varphi_{\mathrm{MZI} 4}}{2}\right) \cos ^{2}\left(\alpha L_{\mathrm{DC} 2}\right)+ \\
\sin ^{2}\left(\frac{\Delta \varphi_{\mathrm{MZI3}}}{2}\right) \cos ^{2}\left(\alpha L_{\mathrm{DC} 3}\right)
\end{array}\right] \sin ^{2}\left(\frac{\Delta \varphi_{\mathrm{MZI6}}}{2}\right) .
$$

Thus, overall output of even parity generator (EPG) 
can be written as $\mathrm{OUT}_{\mathrm{EPG}}=m_{2}+m_{3}+m_{5}+m_{8}$, since there is no signal at output ports for the rest of min-terms.
The timing diagram of even parity generator through MATLAB is presented in Fig. 5, which is verified by its truth table given in Table 1 .

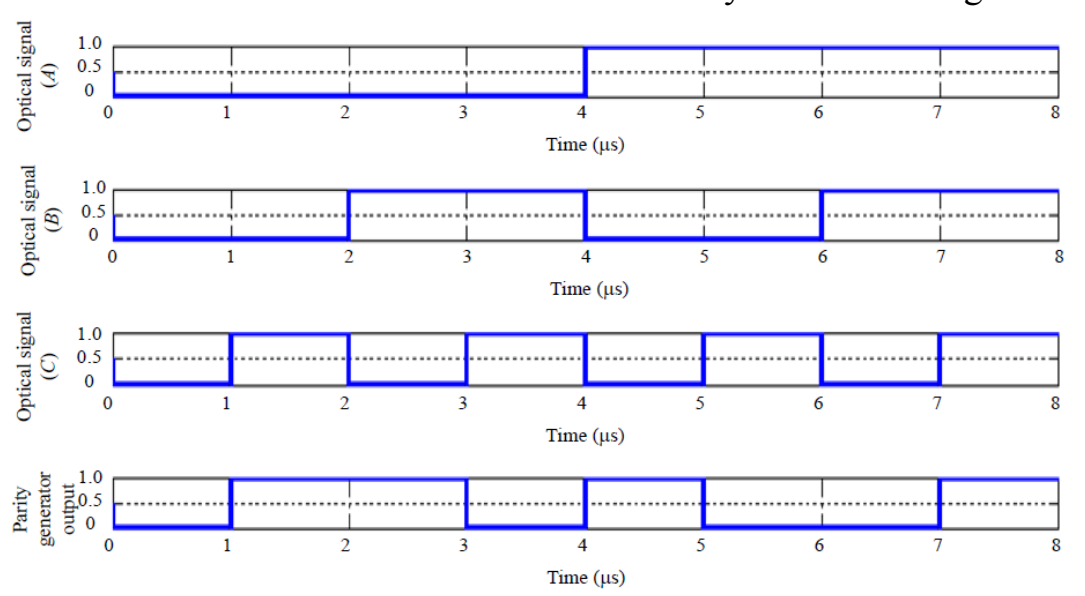

Fig. 5 Timing diagram of even parity generator through MATLAB.

In Fig. 5, the first three rows present the timing sequence for input optical signals, and the fourth row presents the timing signals for the output of parity generator. In the timing diagram, signals in the fourth row with higher magnitudes represent the presence of signal at output, while signals with lower magnitude show absence of signal.

\subsection{Odd parity generator}

Figure 6 shows a digital circuit and K-map of a three-bit-odd-parity generator, and Table 2 presents the truth table of odd parity generator. The design of an odd-parity generator is obtained by cascading seven MZIs and five DCs as shown in Fig. 7.
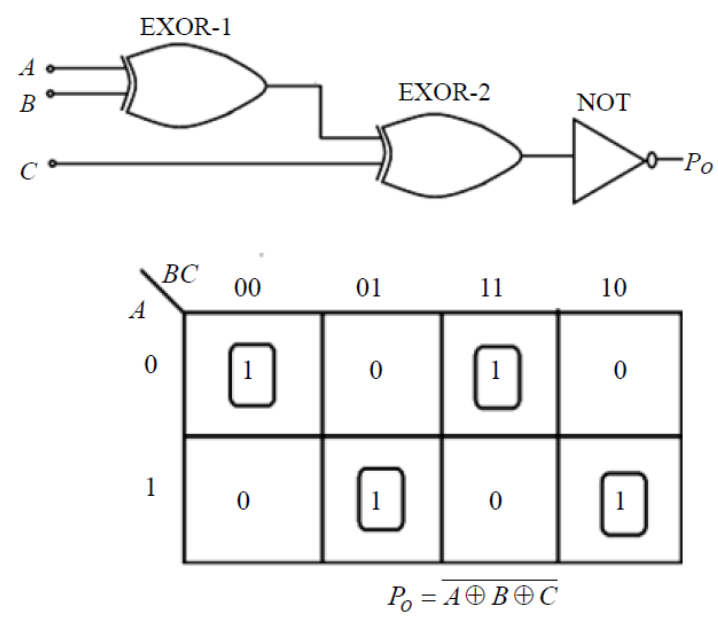

Fig. 6 Digital circuit and K-map of a three-bit-odd-parity generator.

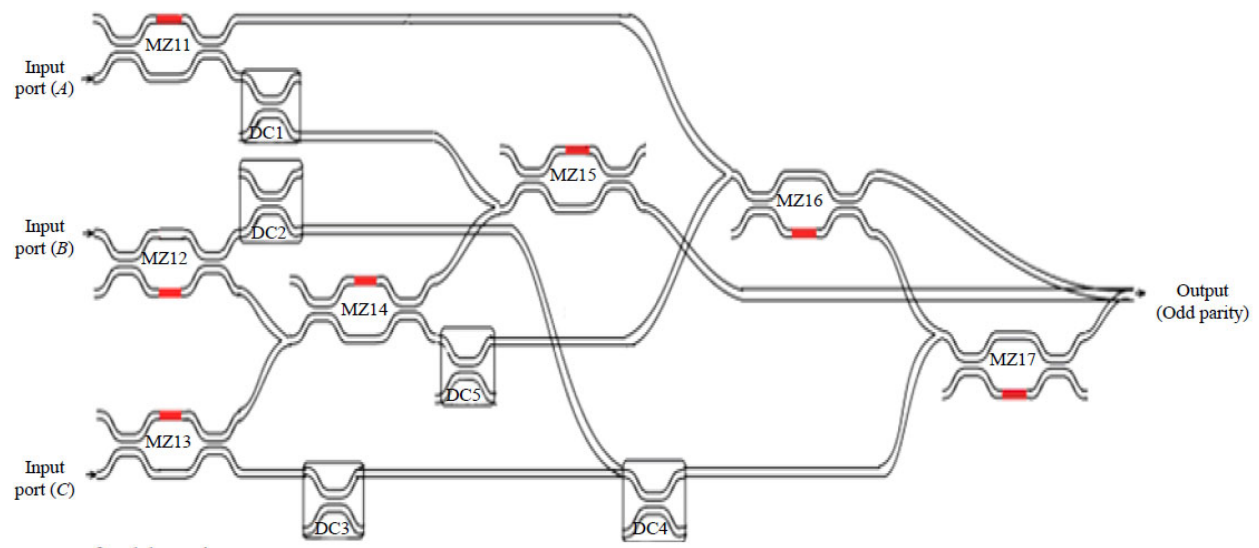

Fig. 7 Block diagram of odd parity generator.

Input optical signals are given at the second, first respectively. $\mathrm{DC} 1$ and $\mathrm{DC} 2$ are connected at the and second input ports of MZI1, MZI2, and MZI3, second and first output ports of MZI1 and MZI2, 
respectively. The second and first output ports of MZI2 and MZI3 are cobbled together to feed the MZI4 with optical signals at its second input port, while the second and first output ports of DC1 and MZI4 are combined together to feed MZI5 at its second input port. To feed MZI6 at its first input port, the first output ports of MZI1 and DC5 (which is connected at the second output port of MZI4) are combined together. The second and first output ports of DC2 and DC3 (which is connected at the second output port of MZI3) are combined together to feed DC4, whose first output port is combined with the second output port of MZI6 to feed the MZI7. Finally, to get the output of odd parity generator, the first output ports of MZI6 and MZI7 are combined together with the second output port of MZI5.

The mathematical expression for outputs of odd parity generator circuit using (1)-(3) can be written as

$$
\begin{aligned}
& m_{1}=\left\{\begin{array}{l}
\cos ^{2}\left(\frac{\Delta \varphi_{\mathrm{MZI}}}{2}\right)+\left[\begin{array}{l}
\cos ^{2}\left(\frac{\Delta \varphi_{\mathrm{MZI} 2}}{2}\right) \\
\cos ^{2}\left(\frac{\Delta \varphi_{\mathrm{MZI3}}}{2}\right)
\end{array}\right] \times \\
\sin ^{2}\left(\frac{\Delta \varphi_{\mathrm{MZI}}}{2}\right) \sin ^{2}\left(\alpha L_{\mathrm{DC} 5}\right)
\end{array}\right\} \sin ^{2}\left(\frac{\Delta \varphi_{\mathrm{MZI}}}{2}\right) \\
& m_{2}=\left[\begin{array}{l}
\cos ^{2}\left(\frac{\Delta \varphi_{\mathrm{MZI} 1}}{2}\right) \cos ^{2}\left(\frac{\Delta \varphi_{\mathrm{MZI}}}{2}\right)+ \\
\sin ^{2}\left(\frac{\Delta \varphi_{\mathrm{MZI} 3}}{2}\right) \sin ^{2}\left(\alpha L_{\mathrm{DC} 3}\right) \sin ^{2}\left(\alpha L_{\mathrm{DC} 4}\right)
\end{array}\right] \times \\
& \cos ^{2}\left(\frac{\Delta \varphi_{\mathrm{MZI} 7}}{2}\right)+\cos ^{2}\left(\frac{\Delta \varphi_{\mathrm{MZI} 2}}{2}\right) \times \\
& \cos ^{2}\left(\frac{\Delta \varphi_{\mathrm{MZI} 4}}{2}\right) \cos ^{2}\left(\frac{\Delta \varphi_{\mathrm{MZI5}}}{2}\right) \\
& m_{3}=\left[\begin{array}{l}
\cos ^{2}\left(\frac{\Delta \varphi_{\mathrm{MZI}}}{2}\right) \cos ^{2}\left(\frac{\Delta \varphi_{\mathrm{MZI} 6}}{2}\right)+ \\
\sin ^{2}\left(\frac{\Delta \varphi_{\mathrm{MZI} 2}}{2}\right) \sin ^{2}\left(\alpha L_{\mathrm{DC} 2}\right) \sin ^{2}\left(\alpha L_{\mathrm{DC} 4}\right)
\end{array}\right] \times \\
& \cos ^{2}\left(\frac{\Delta \varphi_{\mathrm{MZI} 7}}{2}\right)+\cos ^{2}\left(\frac{\Delta \varphi_{\mathrm{MZI} 3}}{2}\right) \times \\
& \cos ^{2}\left(\frac{\Delta \varphi_{\mathrm{MZI} 4}}{2}\right) \cos ^{2}\left(\frac{\Delta \varphi_{\mathrm{MZI} 5}}{2}\right)
\end{aligned}
$$

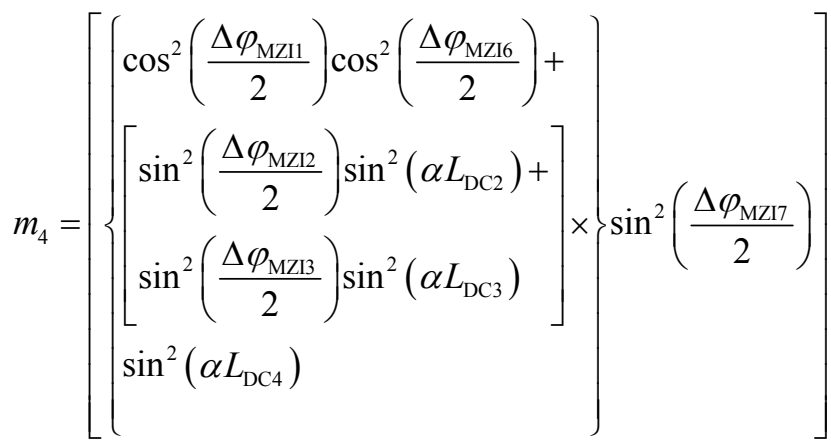

$$
\begin{aligned}
& m_{5}=\sin ^{2}\left(\frac{\Delta \varphi_{\mathrm{MZI} 1}}{2}\right) \cos ^{2}\left(\alpha L_{\mathrm{DC} 1}\right) \cos ^{2}\left(\frac{\Delta \varphi_{\mathrm{MZI}}}{2}\right)+ \\
& {\left[\cos ^{2}\left(\frac{\Delta \varphi_{\mathrm{MZI} 2}}{2}\right)+\cos ^{2}\left(\frac{\Delta \varphi_{\mathrm{MZI} 3}}{2}\right)\right] \times} \\
& \sin ^{2}\left(\frac{\Delta \varphi_{\mathrm{MZI} 4}}{2}\right) \sin ^{2}\left(\alpha L_{\mathrm{DC} 5}\right) \times \\
& \cos ^{2}\left(\frac{\Delta \varphi_{\mathrm{MZI} 6}}{2}\right) \cos ^{2}\left(\frac{\Delta \varphi_{\mathrm{MZI} 7}}{2}\right) \\
& m_{6}=\left[\begin{array}{l}
\sin ^{2}\left(\frac{\Delta \varphi_{\mathrm{MZI} 1}}{2}\right) \cos ^{2}\left(\alpha L_{\mathrm{DC} 1}\right)+ \\
\cos ^{2}\left(\frac{\Delta \varphi_{\mathrm{MZI} 2}}{2}\right) \cos ^{2}\left(\frac{\Delta \varphi_{\mathrm{MZI} 4}}{2}\right)
\end{array}\right] \sin ^{2}\left(\frac{\Delta \varphi_{\mathrm{MZI} 5}}{2}\right)+ \\
& \sin ^{2}\left(\frac{\Delta \varphi_{\mathrm{MZI3}}}{2}\right) \sin ^{2}\left(\alpha L_{\mathrm{DC} 3}\right) \times \\
& \sin ^{2}\left(\alpha L_{\mathrm{DC} 4}\right) \cos ^{2}\left(\frac{\Delta \varphi_{\mathrm{MZI} 7}}{2}\right) \\
& m_{7}=\left[\begin{array}{l}
\sin ^{2}\left(\frac{\Delta \varphi_{\mathrm{MZI} 1}}{2}\right) \cos ^{2}\left(\alpha L_{\mathrm{DC} 1}\right)+ \\
\cos ^{2}\left(\frac{\Delta \varphi_{\mathrm{MZI} 3}}{2}\right) \cos ^{2}\left(\frac{\Delta \varphi_{\mathrm{MZI} 4}}{2}\right)
\end{array}\right] \times \\
& \sin ^{2}\left(\frac{\Delta \varphi_{\mathrm{MZI} 5}}{2}\right)+\sin ^{2}\left(\frac{\Delta \varphi_{\mathrm{MZI} 2}}{2}\right) \sin ^{2}\left(\alpha L_{\mathrm{DC} 2}\right) \times \\
& \sin ^{2}\left(\alpha L_{\mathrm{DC} 4}\right) \cos ^{2}\left(\frac{\Delta \varphi_{\mathrm{MZI} 7}}{2}\right) \\
& m_{8}=\sin ^{2}\left(\frac{\Delta \varphi_{\mathrm{MZI1}}}{2}\right) \cos ^{2}\left(\alpha L_{\mathrm{DC} 1}\right) \cos ^{2}\left(\frac{\Delta \varphi_{\mathrm{MZI5}}}{2}\right)+ \\
& {\left[\begin{array}{l}
\sin ^{2}\left(\frac{\Delta \varphi_{\mathrm{MZI} 2}}{2}\right) \sin ^{2}\left(\alpha L_{\mathrm{DC} 2}\right)+ \\
\sin ^{2}\left(\frac{\Delta \varphi_{\mathrm{MZI} 3}}{2}\right) \sin ^{2}\left(\alpha L_{\mathrm{DC} 3}\right)
\end{array}\right] \sin ^{2}\left(\alpha L_{\mathrm{DC} 3}\right) \times} \\
& \cos ^{2}\left(\frac{\Delta \varphi_{\mathrm{MZI7}}}{2}\right)+\sin ^{2}\left(\frac{\Delta \varphi_{\mathrm{MZI2}}}{2}\right) \sin ^{2}\left(\alpha L_{\mathrm{DC} 2}\right) \times \\
& \sin ^{2}\left(\alpha L_{\mathrm{DC} 4}\right) \cos ^{2}\left(\frac{\Delta \varphi_{\mathrm{MZI} 7}}{2}\right)
\end{aligned}
$$


Table 2 Truth table of odd parity generator.

\begin{tabular}{ccccc}
\hline \multirow{2}{*}{ Min-terms } & \multicolumn{3}{c}{ Input signals } & Output signals \\
\cline { 2 - 5 } & $A$ & $B$ & $C$ & $P_{O}$ \\
\hline$m_{1}$ & 0 & 0 & 0 & 1 \\
$m_{2}$ & 0 & 0 & 1 & 0 \\
$m_{3}$ & 0 & 1 & 0 & 0 \\
$m_{4}$ & 0 & 1 & 1 & 1 \\
$m_{5}$ & 1 & 0 & 0 & 0 \\
$m_{6}$ & 1 & 0 & 1 & 1 \\
$m_{7}$ & 1 & 1 & 0 & 1 \\
$m_{8}$ & 1 & 1 & 1 & 0 \\
\hline
\end{tabular}

Thus, the overall output of odd parity generator (OPG) is written as $\mathrm{OUT}_{\mathrm{OPG}}=m_{1}+m_{4}+m_{6}+m_{7}$, since there is no optical signal at the output port for the rest of min-terms.

The timing diagram of the odd parity generator through MATLAB is presented in Fig. 8, which is verified by its truth table given in Table 2. In Fig. 8, the first three rows represent the timing signals for input signals, and the fourth row represents the timing signals for the generated output of the odd parity generator, which is truly matched with its truth table. In timing diagram at the fourth row, timing signals with low and high magnitudes only represent the presence and absence of optical signals at the desired output.

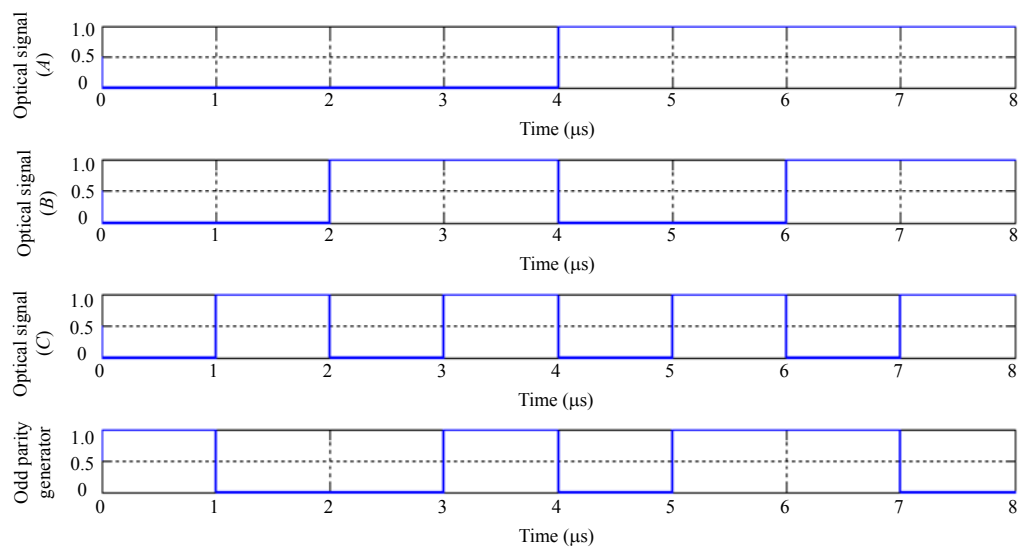

Fig. 8 Timing diagram of odd parity generator.

\section{Simulation results using FDTD}

The finite difference time domain (FDTD) method has been used for analysis of propagation of optical signals through the design of parity generator circuits, and their verifications are done by using MATLAB. The continuous wave $(\mathrm{CW})$ source is used to provide the input signals with Gaussian distribution under the transverse magnetic (TM) polarization at the wavelength of $1550 \mathrm{~nm}$. The Gaussian distribution of signals spectrum is very useful because the interference occurs with side lobes of spectrum, while the maximum information is transmitted on the peak lobe which remains unaffected. For the simulation of the proposed device, perfect matched layers are considered as boundary layers for all interfaces of metal and dielectric with $1 \mathrm{e}^{-12}$ as reflection coefficient. The half width of input source is $0.39 \mu \mathrm{m}$ within the mesh size of $\Delta x=0.021 \mu \mathrm{m}$ and $\Delta z=0.051 \mu \mathrm{m}$, under the perfect matched layer (PML) as boundary conditions for all interfaces of metal and dielectric.

\subsection{Simulation results of even parity generator}

The simulation result of propagation of light through the even parity generator with combination of all possible input signals is presented in Fig. 9.

(1) Case I: $A=0, B=0, C=0$

In this case, $A=B=C=0$ means all three input ports are fed with optical signals having low input power, and thus by following the phenomenon of SPM, these signals are obtained at the first output ports of MZI1, MZI2, and MZI3. Hence, there is no signal at the output port of the even parity generator as shown in Fig.9, which is truly matched with its truth table given in Table 1.

(2) Case II: $A=0, B=0, C=1$

Here, $A=B=0$ means low-power signals are 
Lokendra SINGH et al.: Modeling of All-Optical Even and Odd Parity Generator Circuits Using Metal-Insulator-Metal Plasmonic 189 Waveguides

incident at the second input ports of MZI1 and MZI2, and by obeying the principle of SPM, they arrive at the first output ports of the same MZIs. While $C=1$ means optical high-power signals of high power are provided at the second input port of MZI3 and due to XPM, they arrive at the second output port, where they propagate through DC3 and get split into two equal parts. Then MZI5 gets input optical signals at its first input port from the first output port of DC3, where they obey the laws of SPM due to its low power and are obtained at the second output port of MZI5, which is considered as the output port of EPG. Signals from the second output port of DC3 propagate through MZI6 by obeying the laws of SPM and arrive at its second output port. Thus, in this case, optical signals arrive at the desired output port of EPG due to optical signals of high power at input of MZI3 as shown in Fig. 9.

(3) Case III: $A=0, B=1, C=0$

Here, $A=C=0$ means the low-power optical signala are given at the second input ports of MZI1 and MZI3. $B=1$ means optical high-power signals are incident at the second input port of MZI2. Thus, output optical signals arrive at the desired output port of EPG due to the presence of optical high-power signals at MZI2, which is truly matched with the truth table given in Table 1.

(4) Case IV: $A=0, B=1, C=1$

In this case, $A=0$ means low-power signals are given at the second input port of MZI1. $B=C=1$ means signals of high power are incident at second input ports of MZI2 and MZI3. Hence, in this case output of EPG is zero as shown in Fig. 9.

(5) Case V: $A=1, B=0, C=0$

Here, $A=1$ means high-power signals are given at the second input port of MZI1. $B=C=0$ means low-power signals are given at second input ports of MZI2 and MZI3. In this case, optical signals arrive at the desired output port of EPG, due to the presence of high-powers signal at the second input port of MZI1 (as shown in Fig. 9).

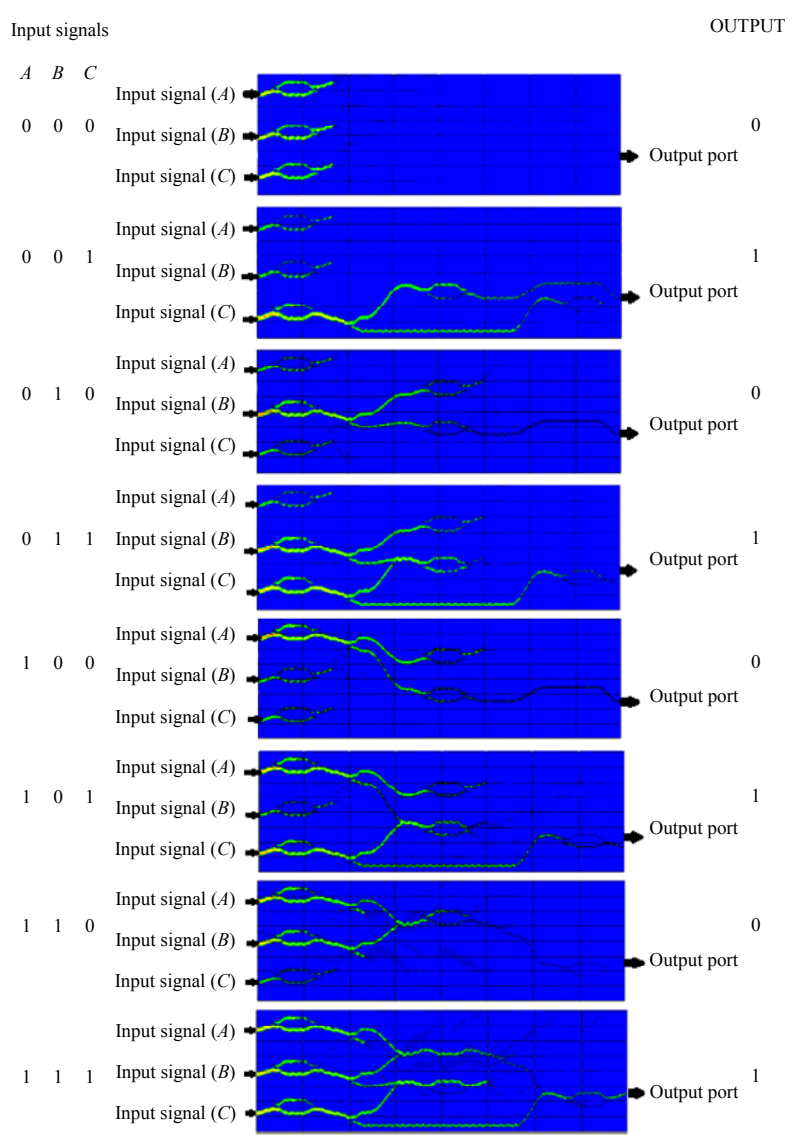

Fig. 9 Propagation of the optical signals through the even parity generator circuit for all possible combinations of input signals obtained through the FDTD method.

(6) Case VI: $A=1, B=0, C=1$

Here, $A=C=1$ means high-power signals are given at the second input ports of MZI1 and MZI3. $B=0$ means low-power signals are given at the second input port of MZI2. In this case, there is no optical signal at the desired output port of EPG, which is truly matched with the truth table shown in Table 1.

(7) Case VII: $A=1, B=1, C=0$

In this case, $A=B=1$ means high-power signals are given at the second input ports of MZI1 and MZI2. $C=0$ means low-power signals are given at the second input port of MZI3. Thus, in this case there is no optical signal at the desired output port of EPG.

(8) Case VIII: $A=1, B=1, C=1$

In this case, $A=B=C=1$ means high-power signals are given at the second input ports of MZI1, MZI2, and MZI3. Hence, in this case optical signals 
are obtained at the desired port of EPG, which is truly matched with its truth table.

\subsection{Simulation results odd parity generator}

The propagation of light through its model with all possible combinations of input signals is presented in Fig. 10.

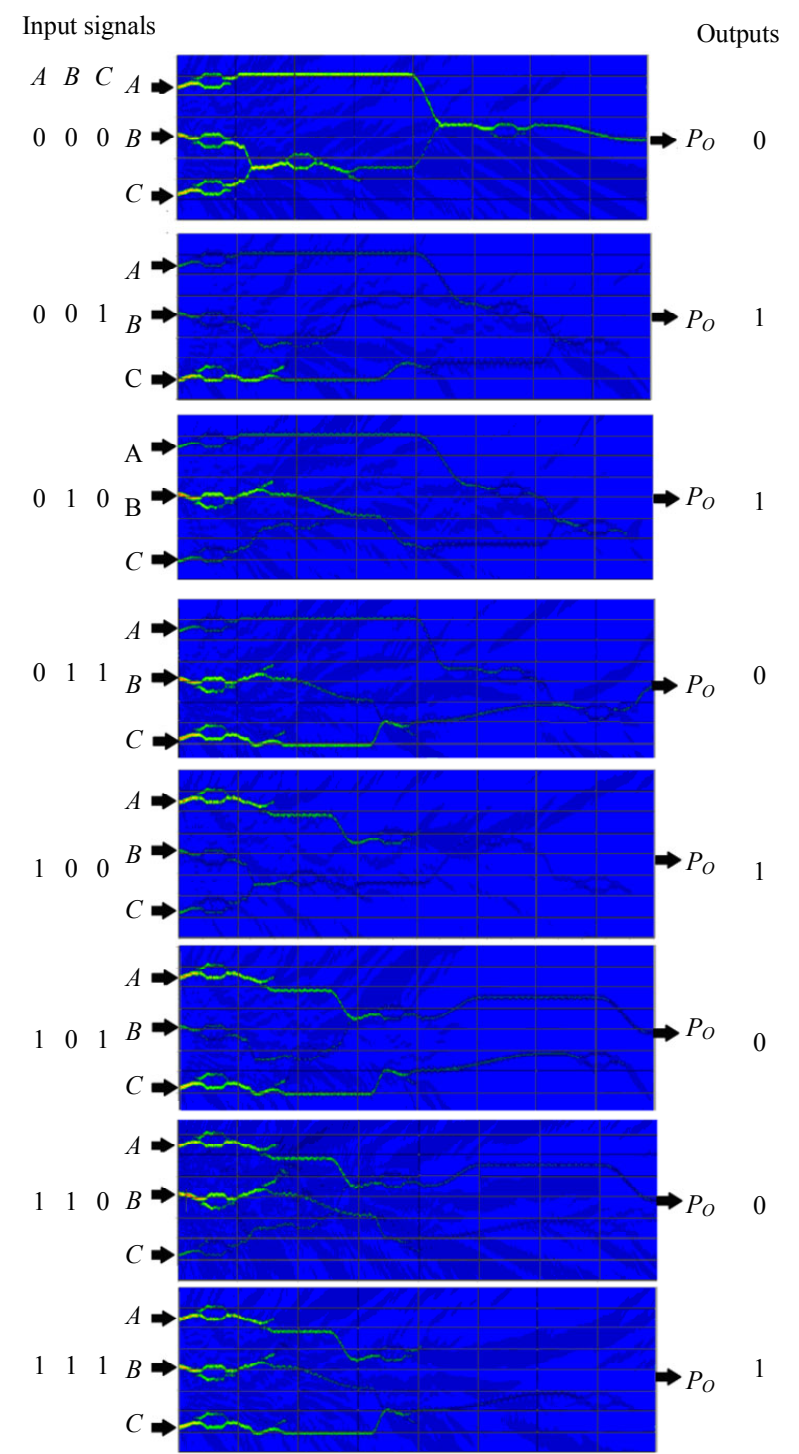

Fig. 10 Propagation of optical signals through the model of the odd parity generator for all combinations of inputs obtained through the FDTD method.

(1) Case I: $A=0, B=0, C=0$

In this case, $A=B=C=0$ means low-power signals are given at the second, first, and second input port of MZI1, MZI2, and MZI3, respectively, and by obeying the phenomenon of SPM the signals are obtained at the first, second, and first output ports of same MZIs. Then signals from the second and first output port of MZI2 and MZI3 are combined together to feed the MZI4 at its second input port, and by following the laws of XPM they arrive at the second output port of same MZI, where they propagate through DC5 and get equally split. Then signals from the first output ports of MZI1 and DC5 are combined together to feed MZI6 at its first input port, and due to its high power they obey the laws of XPM and arrive at the first output port of MZI6, which is considered as the output port for odd parity generator. Thus, in this case an optical signal arrives at the desired output port of OPG, which is truly matched with its truth table given in Table 2 .

(2) Case II: $A=0, B=0, C=1$

Here, $A=B=0$ means low-power signals are given at the second and first input ports of MZI1 and MZI2, where they obey the phenomenon of SPM and arrive at the first and second output ports of same MZI. Then signals from the first output port of MZI1 propagate through MZI6 and due to the low power they arrive at the second output port due to SPM. While signals from the second output port of MZI2 are given to the second input port of MZI4, and after travelling through it signals arrive at its first output port due to the low power. Then MZI5 gets optical signals at its second input port from the first output port of MZI4, and after propagating through MZI5, the signals arrive at first output ports of same MZI. $C=1$ means high power signals are given at the second input port of MZI3 and arrive at the second output port due to XPM, where they propagate through DC3 and get equally split. Further, signals from the first output port of DC3 is again split by DC4, and then signals from the second and first output ports of MZI6 and DC4 are cobbled together to feed MZI7 at its first input port. After propagating through MZI7, signals will appear at its second output port due to the low power. Thus in this case, there is no signal at desired output port of OPG.

(3) Case III: $A=0, B=1, C=0$

Here, $A=C=0$ means signals with low powers are 
incident at the second input ports of MZI1 and MZI3. $B=1$ means high-power signals are given at the first input port of MZI2. Thus, in this case there is no optical signal at the desired output port of OPG.

(4) Case IV: $A=0, B=1, C=1$

Here, $A=0$ means the second input port of MZI1. $B=C=1$ means high intensity signals are given at the first and second input ports of MZI2 and MZI3. Hence, in this case optical signals are obtained at the desired output port of OPG, which is truly matched with its truth table.

(5) Case V: $A=1, B=0, C=0$

In this case, $A=1$ means signals with high power are given at the second input port of MZI1. $B=C=0$ means low-power signals are given at the first and second input ports of MZI2 and MZI3. Thus, in this case there is no optical signal at desired output port of OPG.

(6) Case VI: $A=1, B=0, C=1$

Here, $A=C=1$ means the second input ports of MZI1 and MZI3 are fed with high-power signals. $B=0$ means low-power signals are incident at the first input port of MZI2. Thus, in this case optical signals arrive at the desired output port of OPG, which is truly matched with the truth table given in Table 2.

(7) Case VII: $A=1, B=1, C=0$

Here, $A=B=1$ means high-power signals are given at the second and first input ports of MZI1 and MZI2. $C=0$ means low-power signals are fed at the second input port of MZI3. Finally, output signals approach to the port which is assigned as output port for OPG.

(8) Case VIII: $A=1, B=1, C=1$

Here, as $A=B=C=1$ means high-power signals are given at the second, first, and second input ports of MZI1, MZI2, and MZI3. Thus, in this case there is no optical signal at desired output port of OPG.

\section{Conclusions}

In this paper, models of even and odd parity generator are projected by using MIM plasmonic waveguides due to their enormous capability of confining the surface plasmons up to a deep subwavelength scale. The concept of nonlinear MZI using MIM plasmonic waveguides has been used to cascade the desired structures. The proposed concept of cascading the MZIs is useful for integrating the all-optical devices. The circuits of parity generators are mainly used for error detection and correction in optical communication networks.

Open Access This article is distributed under the terms of the Creative Commons Attribution 4.0 International License (http://creativecommons.org/licenses/by/4.0/), which permits unrestricted use, distribution, and reproduction in any medium, provided you give appropriate credit to the original author(s) and the source, provide a link to the Creative Commons license, and indicate if changes were made.

\section{References}

[1] W. Wei, X. Zhang, and X. Ren, “Asymmetric hybrid plasmonic waveguide with centimetric scale propagation length under subwavelength confinement for photonic components," Nanoscale Research Letters, 2014, 9(1): 1-8.

[2] W. L. Barnes, A. Dereux, and W. E. Thomas, "Surface plasmon subwavelength optics," Nature, 2003, 424(6950): 824-830.

[3] D. K. Gramotnev and S. I. Bozhelvonyi, "Plasmonics beyond the diffraction limit," Nature Photonics, 2010, 4(2): 83-91.

[4] Y. Chen and H. Ming, "Review of surface plasmon resonance and localized surface plasmon resonance sensor," Photonic Sensors, 2012, 2(1): 37-49.

[5] G. Veronis and S. H. Fan, "Guided subwavelength plasmonic mode supported by a slot in a thin metal film," Optics Letters, 2005, 30(24): 3359-3361.

[6] L. Liu, H. Zhanghua, and H. Sailing, "Novel surface plasmon waveguide for high integration," Optics Express, 2015, 13(17): 6645-6650.

[7] A. Boltasseva, S. V. Valentyn, B. N. Rasmus, M. Esteban, G. R. Sergio, and I. B. Sergey, "Triangular metal wedges for subwavelength plasmon polariton guiding at telecom wavelengths," Optics Express, 2008, 16(8): 5252-5260.

[8] A. Kumar, J. Gosciniak, V. S. Volkov, S. Papaioannou, D. Kalavrouziotis, K. Vyrsokinos, et al., "Dielectric-loaded plasmonic waveguide components: going practical," Laser \& Photonics Reviews, 2013, 7(6): 938-951. 
[9] W. L. Barnes, A. Dereux, and T. W. Ebbeen, "Surface plasmon subwavelength optics," Nature, 2003, 424(6950): 824-830.

[10] R. Charbonneau, N. Lahoud, G. Mattiussi, and P. Berini, "Demonstration of integrated optics elements based on long-ranging surface plasmon polaritons," Optics Express, 2005, 13(3): 977-984.

[11] L. Liu, Z. Han, and S. He, "Novel surface plasmon waveguide for high integration," Optics Express, 2005, 13(17): 6645-6650.

[12] J. A. Dionne, L. A. Sweatlock, H. A. Atwater, and A. Polman, "Plasmon slot waveguides: towards chip-scale propagation with subwavelength scale localization," Physical Review B, 2006, 73(3): 035407-9.

[13] G. Veronis and S. Fan, "Modes of subwavelength plasmonic slot waveguides," Journal of Lightwave Technology, 2007, 25(9): 2511-2521.

[14] F. Lou, Z. Wang, D. Dai, L. Tylen, and L. Wousnski, "Experimental demonstration of ultra-compact directional couplers based on silicon hybrid plasmonic waveguides," Applied Physics Letters, 2012, 100(24): 241105-1-241105-4.

[15] M. Z. Alam, J. N. Caspers, J. S. Aitchison, and M. Mojahedi, "Compact low loss and broadband hybrid plasmonic directional coupler," Optics Express, 2013, 21(13): 16029-16034.

[16] D. K. Gramotnev, K. C. Vernon, and D. F. P. Pile, "Directional coupler using gap plasmon waveguides," Applied Physics B, 2008, 93(1): 99-106.

[17] Q. Li, Y. Song, G. Zhou, Y. Su, and M. Qiu, "Asymmetric plasmonic-dielectric coupler with short coupling length, high extinction ratio, and low insertion loss," Optics Letters, 2010, 35(19): 3153-3155.

[18] P. Jia, G. Fang, and D. Wang, "Characterization of miniature fiber-optic Fabry-Perot interferometric sensors based on hollow silica tube," Photonic Sensors, 2016, 6(3): 193-198.
[19] M. A. Bader, G. Marowsky, A. Bahtiar, K. Koynov, C. Bubeck, H. Tillmann, et al., "Poly ( $p$-phenylenevinylene) derivatives: new promising materials for nonlinear all-optical waveguide switching," Journal of the Optical Society of America B, 2002, 19(9): 2250-2262.

[20] S. Kumar, Chanderkanta, and A. Amphawan, "Design of parity generator and checker circuit using electro-optic effect of Mach-Zehnder interferomenters," Optics Communications, 2016, 364: 195-224.

[21] L. Wang, Y. Wang, C. Wu, and F. Wang, "All-optical flip-flop based on coupled SOA-PSW," Photonic Sensors, 2016, 6(4): 366-371.

[22] X. S. Christina and A. P. Kabilan, "Design of optical logic gates using self-collimated beams in $2 d$ photonic crystal," Photonic Sensors, 2012, 2(2): $173-179$.

[23] J. A. Pereda, A. Vegas, and A. Prieto, “An improved compact 2D full-wave FDTD method for general guided wave structures microwave," Microwave \& Optical Technology Letters, 2003, 38(4): 331-335.

[24] S. Kumar, L. Singh, S. K. Raghuwanshi, and N. K. Chen, "Design of full-adder and full-subtractor using metal-insulator-metal plasmonic waveguides" Plasmonics, 2016, 11: 1-11.

[25] S. Kumar, L. Singh, and S. Swarnakar, "Design of one bit magnitude comparator using nonlinear plasmonic waveguide," Plasmonics, 2016, 11: 1-7.

[26] S. Kumar and L. Singh, "Proposed new approach to design all optical AND gate using plasmonic based Mach-Zehnder interferometer for high speed communication," SPIE, 2016, 9884: 98842D.

[27] S. K. Raghuwanshi, A. Kumar, and S. Kumar, " $1 \times 4$ signal router using three Mach-Zhender interferometers," Optical Engineering, 2013, 52(3): 035002-1-035002-9.

[28] G. P. Agrawal, Nonlinear fiber optics. Salt Lake City: Academic Press, 2006. 